\title{
Factores de riesgo y estrategias de prevención para el desarrollo de estrechez uretral de origen iatrogénico: Papel del urólogo
}

\section{Risk Factors and Prevention Strategies for the Development of Urethral Stricture of Iatrogenic Origin: Role of the Urologist}

\author{
Andres Goméz Hoyos ${ }^{1}$ Federico Gaviria Gil ${ }^{1}$ \\ ${ }^{1}$ Urólogo, Universidad CES, Medellín, Colombia. \\ Address for correspondence Andres Goméz Hoyos, MD, Urólogo, \\ Clínica Universidad CES, Dirección: cra 42 \# 5 sur - 46, Medellín, \\ Urol Colomb 2021;30:66-73. \\ Colombia (e-mail: andresdocmed@gmail.com).
}

\section{Resumen \\ Palabras clave \\ - estrechez uretral \\ - procedimientos quirúrgicos urológicos \\ - cateterismo urinario \\ - cistoscopia \\ - enfermedad iatrogénica}

La estrechez uretral constituye una patología con morbilidad importante en el sexo masculino. Se evidencia en la actualidad un cambio en la frecuencia de las etiologías, con la disminución de causas inflamatorias y una transición hacia la iatrogenia como la más común. Mediante la búsqueda del estado del arte en cuanto a los procedimientos diagnósticos y terapéuticos conocidos como factores asociados a la estrechez uretral iatrogénica, se realizó una revisión narrativa de la literatura con el fin de describir y generar estrategias para su prevención. De los procedimientos terapéuticos que originan la estrechez uretral como complicación, el sondaje vesical es la mayor causa (hasta 34,3\%), seguido de la prostatectomía radical (29,9\%). Una buena técnica de sondaje vesical orientada desde el adecuado entrenamiento del personal disminuye de forma considerable su incidencia. Por otra parte, la adecuada selección de tratamientos y aspectos técnicos en pacientes que requieren el manejo de patologías obstructivas del tracto urinario como la hiperplasia prostática y litiasis u oncológicas como el cáncer de próstata, son unas de las recomendaciones para la prevención de ese trastorno. El entendimiento de los factores de riesgo y la adherencia a las estrategias de prevención descritas buscan disminuir la incidencia de la estrechez uretral de origen iatrogénico.

Urethral stricture is a pathology with significant morbidity in men. There is current evidence of a change in the prevalence of etiologies, with a decrease in inflammatory causes and a transition towards iatrogenesis as the most frequent. Through the search for the state of art in terms of diagnostic and therapeutic procedures known as associated factors to iatrogenic urethral stricture, a narrative review has been performed, in order to describe and recommend strategies for its prevention. Of the therapeutic procedures that cause urethral stricture as a complication, bladder received

August 28, 2020

accepted

November 17, 2020
DOI https://doi.org/

$10.1055 / \mathrm{s}-0040-1722237$.

ISSN 0120-789X.

e ISSN 2027-0119.

\footnotetext{
(c) 2021. Sociedad Colombiana de Urología. All rights reserved. This is an open access article published by Thieme under the terms of the Creative Commons Attribution-NonDerivative-NonCommercial-License, permitting copying and reproduction so long as the original work is given appropriate credit. Contents may not be used for commercial purposes, or adapted, remixed, transformed or built upon. (https://creativecommons.org/ licenses/by-nc-nd/4.0/)

Thieme Revinter Publicações Ltda., Rua do Matoso 170, Rio de Janeiro, RJ, CEP 20270-135, Brazil
} 


\author{
Keywords \\ - urethral stricture \\ - urologic surgical \\ procedures \\ - urinary \\ catheterization \\ - cystoscopy \\ - iatrogenic disease
}

catheterization is the main (up to $34.3 \%$ ), followed by radical prostatectomy (29.9\%). A good bladder catheterization technique guided by adequate training of personnel considerably reduces its incidence. On the other hand, the proper selection of treatments and technical aspects in patients who require the management of obstructive pathologies of the urinary tract such as prostatic hyperplasia and lithiasis or oncological diseases such as prostate cancer, are some of the recommendations for the prevention of this disorder. Understanding risk factors and adherence to the prevention strategies described seek to reduce the incidence of iatrogenic urethral stricture.

\section{Introducción}

La estrechez uretral ha tenido un cambio considerable de etiología, siendo actualmente la iatrogenia, el trauma y la de origen idiopático las causas más frecuentes por encima de las inflamatorias secundarias a infección gonocócicas y no gonocócica. ${ }^{1}$ La iatrogenia representa hasta el $45,5 \%$ de los casos descritos en las últimas series publicadas, ${ }^{2}$ y eso se explica, en gran medida, por las características propias de la uretra masculina tales como su longitud, puntos de fijación, histología e irrigación sanguínea, que la hacen propensa al desarrollo de lesiones durante la instrumentación ${ }^{3}$ (ver Relaciones anatómicas de la uretra). Hoy en día se practican diversos procedimientos diagnósticos y terapéuticos del tracto urinario que involucran la manipulación de la uretra, como la resección transuretral de próstata (RTU-P) y tumores vesicales, el cateterismo uretral, la cistoscopia, el manejo endoscópico de la litiasis, la prostatectomía abierta entre otros, pudiendo preverse en el corto y mediano plazo un incremento significativo tras el desarrollo e implementación de nuevas tecnologías para el tratamiento mínimamente invasivo de la hiperplasia prostática (fotovaporización, enucleación con láser) ${ }^{4}$ así como para el cáncer de próstata localizado (crioterapia, HIFU y otras terapias focales). ${ }^{5}$

El cambio en la etiología de la estrechez uretral que posiciona a la iatrogenia como una de las causas más relevantes, obliga a plantear una mirada crítica al papel activo que juega el urólogo en el desarrollo de esa patología. El objetivo de esta revisión de la literatura, fue describir los factores de riesgo de la estrechez uretral asociados a los procedimientos urológicos más comúnmente empleados y las estrategias recomendadas para su prevención.

\section{Métodos}

La búsqueda de literatura se realizó en las bases de datos electrónicas PubMed, EMBASE, SciELO y Cochrane, mediante las palabras clave: estrechez uretral, procedimientos quirúrgicos urológicos, cateterismo urinario, cistoscopia, enfermedad iatrogénica. No se siguió una estrategia de búsqueda estructurada al tratarse de una revisión narrativa. Sin embargo, la búsqueda abarcó artículos enfocados en la descripción de las causas más frecuentes de estrechez uretral y que influyeran en el desarrollo de estrategias de prevención (ver Flujograma de búsqueda. -Fig. 3).

\section{Relaciones Anatómicas de la Uretra (-Figs. 1 y 2)}

\section{Cateterismo Vesical}

El cateterismo vesical es uno de los procedimientos más comúnmente realizados en pacientes hospitalizados, y se encuentra asociado a una importante morbilidad por su relación con el desarrollo de infección urinaria nosocomial ${ }^{6}$ además de explicar hasta el 34\% de las causas iatrogénicas de la estrechez uretral. ${ }^{7}$ La educación sobre la técnica apropiada de cateterización vesical es el primer paso hacia la prevención de esa lesión. ${ }^{8}$ Desafortunadamente, el personal de salud técnico y profesional involucrado activamente en la elaboración del procedimiento suele poseer un grado variable de destreza para su adecuada realización, siendo en ocasiones insuficiente. Grandes centros de tercer nivel en EE. UU. han reportado

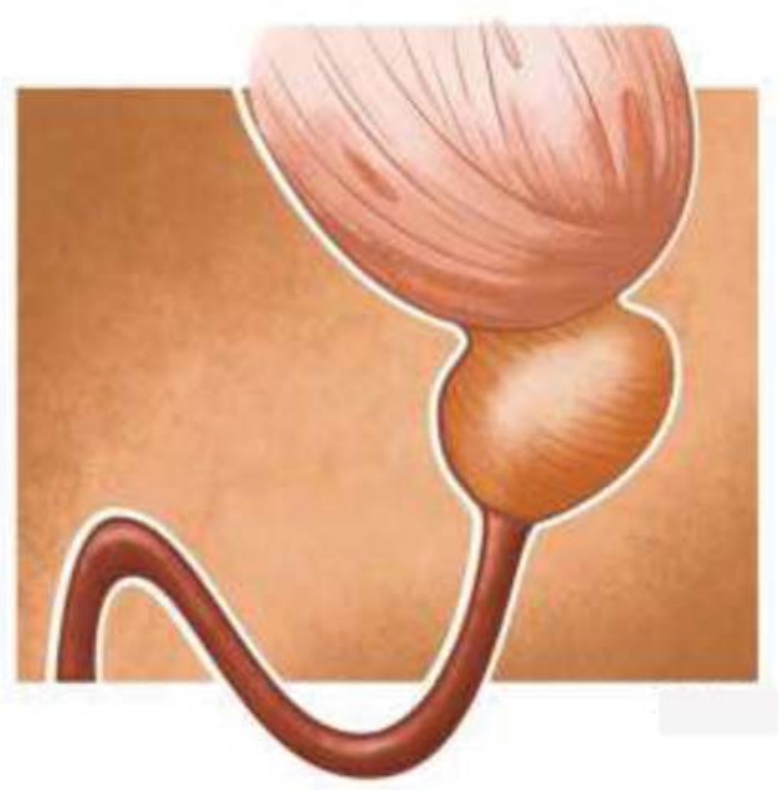

Fig. 1 Anatomía de la uretra masculina. Fuente: Elaboración propia. 


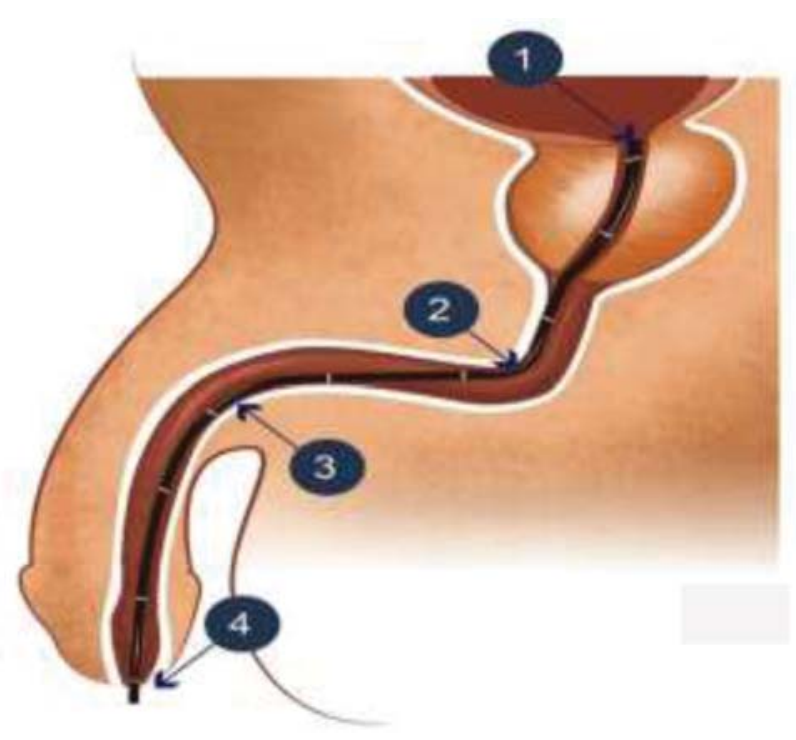

Fig. 2 Relación anatómica con la instrumentación del tracto urinario bajo. Fuente: Elaboración propia.

deficiencias o nulo entrenamiento para el paso de una sonda vesical en el $76 \%$ de los médicos internos de la institución ${ }^{9}$, logrando establecer una relación directa con un incremento del riesgo para el desarrollo de estrechez uretral iatrogénica en ese centro. Es por esa razón, que la implementación de programas para la educación del personal puede disminuir de forma significativa la incidencia de lesiones uretrales, reduciendo la aparición de estrechez secundaria al procedimiento, ${ }^{10}$ sesiones prácticas y el empleo de modelos para instruir sobre los puntos criticos de la técnica en el paso correcto de la sonda vesical tales como la tracción del pene con corrección del ángulo peno escrotal, la adecuada lubricación, paso de la sonda hasta vejiga sin que ésta se devuelva de forma espontánea, así como la verificación de la salida de orina previo a inflar el balón y que este se infle sin resistencia, pueden disminuir la incidencia de lesiones de 3/1.000 a 0.7/ 1.000 casos.

Otra consideración importante en el manejo de la sonda vesical se presenta en situaciones clínicas que requieren su uso por tiempo prolongado, condicionado por patologías funcionales y estructurales del tracto urinario o el caso común del paciente crítico internado en la Unidad de cuidados intensivos (UCI), donde hasta el $90 \%{ }^{2}$ pueden necesitar sondaje con grados variables de duración. En ese escenario específico, cuando se prevén tiempos cortos de uso, sondas de materiales como el látex se encuentran asociadas a una mayor reacción inflamatoria y posterior estrechez de la uretra, por lo que se recomienda el uso de sondas de silicona pura ${ }^{11}$ con calibre reducido. ${ }^{12}$ Medidas alternativas como el uso de colectores externos o cateterismos intermitentes cuando la situación clínica lo permite, así como la colocación de un catéter suprapúbico temporal cuando no son factibles abordajes menos invasivos, pueden ser la mejor opción de manejo. Esa última aproximación, intenta emular los protocolos de UCI en pacientes con ventilación invasiva prolongada llevados a traqueostomía de forma temprana para prevenir el desarrollo de estenosis laringotraqueal. Cualquiera de las medidas anteriormente descritas logra disminuir la aparición de complicaciones como fístulas, absceso escrotal y epididimitis, así como el desarrollo de estrechez de uretra anterior, en ocasiones compleja y con necesidad posterior de procedimientos reconstructivos complejos ${ }^{13,14}$ comparativamente al uso de una sonda uretral permanente.

\section{Manejo Quirúrgico de la Hiperplasia Prostática}

El abordaje quirúrgico de la Hiperplasia prostática benigna (HBP) se realiza mediante diversas técnicas (endoscópica, abierta, laparoscópica, robótica). La clara indicación para su uso en el tratamiento de la obstrucción del tracto urinario inferior, con el fin de disminuir morbilidad y complicaciones asociadas, podría ser la primera recomendación en el papel del urólogo, recordando que permanece como la opción ideal de tratamiento en aquellos pacientes con insuficiencia renal secundaria a Hiperplasia prostática benigna (HPB), infección del tracto urinario recurrente, litiasis vesical, hematuria o retención urinaria y persistencia de síntomas del tracto urinario inferior (LUTS) refractarios al manejo médico no invasivo. $^{4}$

Desde los años 70, la resección transuretral de próstata (RTU-P) para el tratamiento de los LUTS secundarios a HPB ha sido la opción terapéutica más popular ${ }^{15}$ y el estándar de oro para comparar la eficacia, efectividad y seguridad de cualquier otro tratamiento invasivo. ${ }^{4}$ Sin embargo, ese procedimiento rutinario no está libre de complicaciones y confiere un riesgo incrementado para el desarrollo de la estrechez uretral, que dependiendo de la serie evaluada, reporta porcentajes de estrechez de uretra anterior y contractura del cuello vesical (CCV) en el 2,2 a 9,8\% y 0,3 a $9,2 \%$ de los casos respectivamente. ${ }^{16}$

Para el desarrollo de la estrechez secundaria a la RTU-P, se han descrito diversos factores relacionados a ubicaciones específicas de la uretra; la estrechez del meato uretral usualmente ocurre por una inapropiada relación entre el tamaño del resectoscopio y el diámetro del meato uretral, ${ }^{16}$ generando no sólo un componente isquémico de la mucosa, sino también un riesgo incrementado de disrupción al paso forzado, lo que permite la extravasación de orina a la región subepitelial ${ }^{15}$ con la consecuente reacción inflamatoria local y el riesgo de estrechez. En ese escenario, sería prudente la dilatación secuencial mediante Benique, previo al inicio del procedimiento, recordando siempre el empleo de una adecuada técnica atraumática. De igual forma se recomienda el uso de resectoscopios de menor calibre 24 FR cuando se encuentren disponibles.

En cuanto al desarrollo de la estrechez de la uretra bulbar durante la RTU-P con el uso de energía monopolar, se ha descrito un daño potencial en la uretra secundario a un aislamiento insuficiente por el lubricante, que favorece la fuga de la corriente durante la resección. ${ }^{16}$ El lubricante debe aplicarse con cuidado en la uretra y a lo largo del eje del resectoscopio, repitiendo su aplicación en tiempos prolongados de resección y evitando además el empleo de 


\section{Búsqueda por palabras clave}

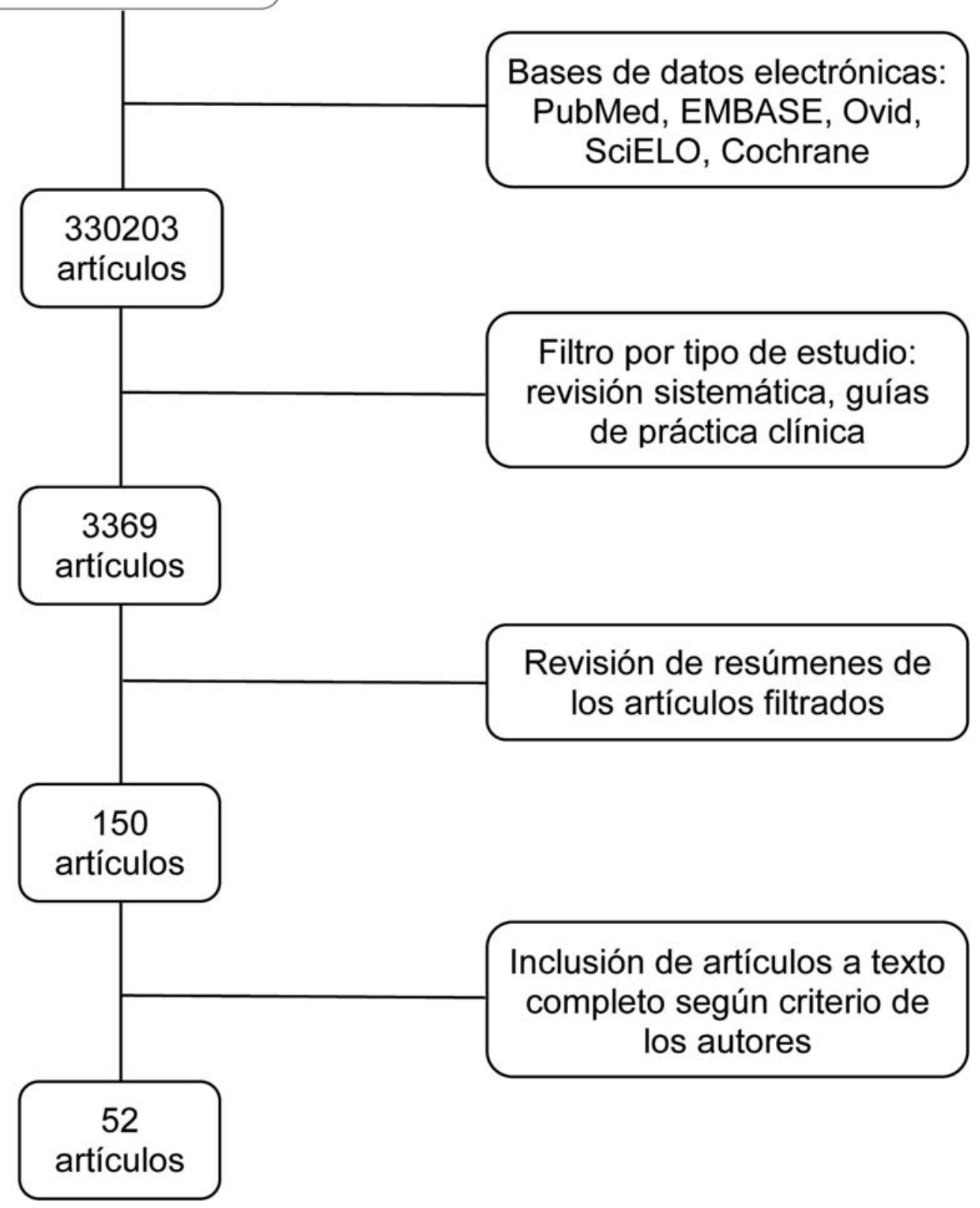

Fig. 3 Flujograma de articulos incluidos.

valores altos de corriente de corte. En cuanto al desarrollo de CCV tras la RTU-P, su aparición se ha relacionado con el tratamiento de próstatas de bajo volumen $(<30 \mathrm{~g})$ donde el daño tisular causado por la electro-resección genera un riesgo incrementado de $\mathrm{CCV}^{17}$ en comparación con próstatas de gran volumen, donde al parecer el cuello vesical se aparta y se protege. ${ }^{18}$ En dicho escenario la incisión transuretral de la próstata (ITP), conservando el cuello vesical, podría ser una alternativa de tratamiento con buenas tasas de éxito, excepto cuando hay crecimiento del lóbulo medio. ${ }^{4}$ Así mismo vale la pena mencionar que el indicar la RTU-P a grandes volúmenes ( $>60 \mathrm{~g}$ ) y con tiempos prolongados de resección ( $>60$ minutos), si bien es técnicamente factible, se vincula con un incremento en la aparición de la estrechez uretral. ${ }^{19}$

Se han propuesto adicionalmente esquemas de manejo médico con inhibidores de la COX-2 en pacientes llevados a RTU P, que, por su efecto antiinflamatorio, podrían modular los procesos de fibrosis postquirúrgica; En un estudio prospectivo, su aplicación en ciclos de 20 días tras el retiro de la sonda uretral se asoció a una marcada disminución en la incidencia de estenosis uretral (17\% vs $0 \%$ ) cuando se comparó con un grupo de control con placebo. $^{20}$ Sin embargo, la evidencia disponible es insuficiente en favor de una recomendación para su uso generalizado. 
En años recientes, nuevos procedimientos endourológicos incluyendo la vaporización fotoselectiva de próstata (PVP) la enucleación de la próstata con Holmium y Thulium laser (HoLEP/ThuLEP), termoterapia transuretral con microondas (TUMT) y la ablación transuretral con aguja (TUNA) ${ }^{16}$, han demostrado su efectividad y seguridad en el tratamiento de la $\mathrm{HPB}^{3}{ }^{3}$ Sin embargo, ninguna de las nuevas técnicas de abordaje transuretral parece disminuir el riesgo de estrechez uretral, reportándose tasas de incidencia de CCV del 1,1 a $7,4 \%$ y del 1,3 a $6 \%$ tras el uso de la vaporización fotoselectiva de próstata y enucleación con láser respectivamente. ${ }^{21-24}$

En cuanto al manejo abierto de la HPB, en series modernas se han reportado tasas de estrechez uretral y CCV, de $3,9 \%$ y $1,9 \%$ respectivamente. ${ }^{25}$ En el caso de la CCV se han vinculado factores como la apertura inapropiada del cuello durante el procedimiento, al igual que fibrosis durante la epitelización de la uretra prostática, por lo que se recomienda una incisión longitudinal en el cuello vesical de forma perpendicular a la capsulotomía transversa para su ampliación y una trigonización adecuada movilizando la mucosa del cuello vesical hacia el piso de la capsula prostática. $^{25,26}$ Especial cuidado se debe tener al momento de la enucleación del ápex, pues una mala técnica puede conllevar un riesgo incrementado de estenosis de la uretra membranosa, con un complicado abordaje reconstructivo futuro por su estrecha relación con el esfínter urinario externo, y un sombrío pronóstico de continencia tras su reconstrucción, en un paciente que ya ha perdido el componente de continencia del cuello vesical tras la cirugía prostática.

\section{Manejo Endoscópico de la Urolitiasis}

Las múltiples modalidades terapéuticas disponibles hacen complejo la elección del tratamiento quirúrgico adecuado y en algunos pacientes, más de una técnica puede ser apropiada. El tratamiento ideal depende de factores específicos del paciente y del cálculo. ${ }^{27}$ Los abordajes mínimamente invasivos, incluyendo los endoscópicos, son hoy en día la primera línea de manejo quirúrgico.

Se han estudiado en detalle las potenciales complicaciones de los abordajes endoscópicos en el tracto urinario superior, hoy en día entendemos varios mecanismos fisiopatológicos relacionados con la sepsis y complicaciones como lesión y estrechez ureteral $(0,2 \% \text { a } 1,4 \%)^{28,29}$ Lamentablemente, no se menciona la incidencia de estrechez uretral secundaria a ese tipo de procedimientos y no hay disponibilidad en la literatura sobre datos concretos de esa asociación.

Como se describió antes, la uretra y sus características la hacen propensa al desarrollo de lesiones iatrogénicas durante la instrumentación, ${ }^{7}$ por lo tanto, abordajes endoscópicos prolongados, producto del abuso de abordajes retrógrados para el tratamiento de cálculos de gran tamaño, son un factor de riesgo para el desarrollo de la estrechez uretral secundaria. Deben considerarse abordajes alternos como el manejo percutáneo en cálculos con volumen litiásico mayor de $2 \mathrm{~cm}$ y cálculos del polo inferior mayores de $1 \mathrm{~cm},{ }^{27}$ más aún teniendo en cuenta la alta recurrencia de la urolitiasis $(40 \%-70 \% \text { a } 5 \text { años })^{30}$ y la necesidad de procedimientos terapéuticos repetidos en un mismo paciente.

\section{Tratamiento del Cáncer de Próstata}

El cáncer de próstata es el principal tumor no cutáneo diagnosticado en el hombre, un verdadero problema de salud pública, con una incidencia de 161,360 casos en Estados Unidos para el 2017 y una supervivencia estimada a 5 años de $98,6 \%{ }^{31}$ Las opciones convencionales para el tratamiento con intención curativa del cáncer de próstata localizado incluyen manejos no invasivos como la vigilancia activa, tratamientos quirúrgicos como la prostatectomía radical mediante abordajes abiertos, laparoscópicos puros o asistidos por robot, diversas técnicas de radioterapia incluidas la radioterapia de haz externo (EBRT), radioterapia tridimensional conformada (3D-CRT), radioterapia de intensidad modulada (IMRT), braquiterapia de baja tasa de dosis (LDR-BT), braquiterapia de alta tasa de dosis (HDR-BT), terapia de haz de protones (PBT) y numerosas terapias ablativas mínimamente invasivas. ${ }^{5,32}$

La fisiopatología implicada en el desarrollo de estrechez uretral secundaria al tratamiento quirúrgico del cáncer de próstata se relaciona con diversos factores que en última instancia comprometen los procesos de cicatrización normal, como edad del paciente, hemorragia excesiva, reconstrucción estrecha del cuello, fuga urinaria, diabetes mellitus, enfermedad coronaria y tabaquismo. ${ }^{33,34}$ Por su parte, la radioterapia predispone al desarrollo de estrechez uretral debido a daño vascular por endarteritis obliterante, apoptosis, formación de tejido fibroso y radionecrosis, ${ }^{35}$ donde la presencia de procedimientos quirúrgicos previos como la RTU-P, incrementan de forma significativa el riesgo de aparición de estrechez secundaria. ${ }^{36}$

La aparición de estenosis uretral relacionada con la prostatectomía radical oscila entre el 1,6\% hasta $29,9 \%$ de los $\operatorname{casos}^{37}$; para aquellas secundarias a radioterapia, varía en gran medida según la modalidad seleccionada. La base de datos CaPSURE evidenció una frecuencia de estenosis de 1,8\% para la braquiterapia, del 1,7\% para la EBRTy del 5,2\% para la radioterapia combinada ${ }^{38}$ y se han reportado incidencias de estenosis después HDR-BT hasta del $11 \%$, lo que refleja una relación dosis dependiente con la aparición de esa estenosis. ${ }^{39}$ De igual forma, terapias ablativas mínimamente invasivas conllevan también a riesgos potenciales de estenosis uretral, como es el caso del ultrasonido enfocado de alta intensidad (HIFU) con tasas de $1,8 \%$ a $36 \% 40,41$ y de la crioterapia primaria de próstata con tasas de hasta $13 \%$ a 4 años. ${ }^{38}$

Es importante mencionar que la primera medida potencial para disminuir la incidencia y complicaciones asociadas con el desarrollo de estrechez uretral secundario al tratamiento del cáncer de próstata consiste en la adecuada elección del tratamiento, eso incluye evitar el sobretratamiento. Sabemos hoy que un número significativo de tumores de próstata presentan un comportamiento indolente $y$ en pacientes bien seleccionados, con enfermedad localizada de bajo riesgo y un adecuado seguimiento es posible ofrecer esquemas de 
Tabla 1 Puntos clave de esta revisión

- La iatrogenia es una causa importante de estrechez uretral en la actualidad.

- Las características anatómicas e histológicas de la uretra la hacen susceptible al desarrollo de la estrechez.

- Adecuada técnica en el paso de la sonda vesical y capacitación del personal de salud; tracción del pene, la adecuada

lubricación, el paso de la sonda hasta el tope, así como la verificación de la salida de orina previo a la insuflación del balón.

- En pacientes que requieren sonda vesical por tiempos cortos considerar materiales como la silicona pura en calibre reducido

(16 Fr) y definir medidas alternativas al uso de sonda uretral permanente: cateterismo uretral intermitente, cistostomía percutánea.

- Selección adecuada de candidatos a RTU de próstata (volúmenes menores a 60 gramos/ tiempo límite 60 minutos), uso de equipos de menor calibre (resectoscopios $24 \mathrm{Fr}$ ) y dilatación atraumática secuencial previo al inicio del procedimiento.

- En el manejo abierto de la HPB, especial cuidado en la enucleación del ápex prostático y una adecuada trigonización en todos los casos.

- No sobre indicar los abordajes retrógrados endoscópicos para el tratamiento de la litiasis renal en cargas litiásicas mayores de $2 \mathrm{~cm}$ o del polo inferior mayores a $1 \mathrm{~cm}$ y evaluar el uso de abordajes alternos (percutáneo).

- En el tratamiento del cáncer de próstata, mediante prostatectomía radical, manejo por grupo con adecuado volumen por año y experiencia, reconstrucción del cuello con calibre de $28 \mathrm{Fr}$ y adecuada oposición mucosa mucosa en la anastomosis; si se emplea radioterapia, recomendar la terapia conformal tridimensional (3D-CRT) y la radioterapia de intensidad modulada (IMRT).

- En el hallazgo de una estrechez uretral durante una cistoscopia, donde no hay un fácil paso de un equipo calibre $16 \mathrm{Fr}$, obliga en la mayoría de los casos a suspender el procedimiento y proceder a la realización de una uretrografia formal para la adecuada caracterización de la estrechez.

vigilancia activa que no representan un incremento significativo de la mortalidad específica por cáncer a 10 años, comparativamente con terapias de intención curativa como radioterapia o prostatectomía radical $^{42}$, estando actualmente recomendada con un grado de evidencia A por diferentes guías de práctica clínica como la Sociedad Americana de Urología (AUA) y la Asociación Europea de Urología (EAU).

En el caso de la prostatectomía radical, las medidas para disminuir la incidencia de estenosis uretral obedecen en primera instancia independiente de la vía, a la experiencia requerida del cirujano que realiza el procedimiento ${ }^{43}$; también modificaciones en la técnica quirúrgica, tales como la reconstrucción del cuello de la vejiga hasta un diámetro de $28 \mathrm{Fr},{ }^{44}$ la adecuada oposición mucosa a mucosa de la uretra y vejiga y la introducción de abordajes laparoscópicos asistidos por robot, que han demostrado en algunas series una disminución de la incidencia de CCV de $2,6 \%$ a $1,4 \%$ comparativamente con abordajes abiertos. ${ }^{43}$ deben ser tenidas en cuenta para mejorar los desenlaces.

En el caso de la radioterapia de haz externo como tratamiento con intención curativa, ésta puede ser administrada mediante el uso de diversas tecnologías, incluida la 3D-CRT y la IMRT, que disminuyen la lesión por radiación de los tejidos circundantes como la uretra, vejiga y recto; la evidencia disponible reporta bajas tasas de estrechez uretral con el uso de esas modalidades, actualmente menores al 3\% 39,45 siendo opciones ideales de tratamiento en pacientes llevados a radioterapia.

\section{Cistoscopia}

La cistoscopia en un procedimiento endoscópico frecuentemente realizado bajo diversas indicaciones como la evaluación diagnóstica de la hematuria, LUTS, fístulas del tracto urinario, malformaciones congénitas, remoción de cuerpos extraños, entre otros. Sea de forma incidental o durante el estudio de sintomatología del tracto urinario inferior, la detección de una estrechez uretral durante una cistoscopia, siempre que no permite el paso fácil de un equipo calibre $16 \mathrm{Fr}$, indica suspender el procedimiento y realizar de forma posterior una uretrografía formal para la adecuada caracterización de la estrechez ${ }^{46}$ y si es grave, la realización de una cistostomía, permitiendo el reposo uretral y posterior estudio para su adecuada caracterización y planeamiento quirúrgico reconstructivo. ${ }^{47-50}$ El papel del urólogo se encuentra en evitar el paso forzado del equipo, el empleo de dilataciones o el uso indiscriminado de otros manejos endoscópicos como la uretrotomía interna endoscópica, contraindicadas en la gran mayoría de escenarios, y que no permiten un adecuado estudio y tratamiento de la patología uretral, derivando en morbilidad, reestenosis y retrasos para un tratamiento quirúrgico definitivo. ${ }^{51,52}$

\section{Conclusiones}

La uretra es la puerta de abordaje para el diagnóstico y tratamiento de múltiples patologías del tracto urinario y dadas sus características anatómicas, es muy propensa al daño con la instrumentación. Si bien diversos artículos tratan los factores de riesgo y estrategias de prevención para el desarrollo de estrechez iatrogénica, suelen ser el resultado de estudios observacionales o casi experimentales no aleatorizados. Bienvenidas son las nuevas tecnologías menos invasivas que han revolucionado el manejo de múltiples patologías del tracto urinario (HPB, urolitiasis, cáncer de próstata), y tanto de su uso racional como adecuada indicación, dependerá en gran medida la disminución de las complicaciones asociadas, incluyendo el desarrollo de estrechez uretral. Por último, mencionamos los puntos clave de esta revisión que resumen las ideas más importantes. (-Tabla 1)

Conflicto de Intereses

Los autores declaran no tener ningún conflicto de intereses. 


\section{Referencias}

1 Fenton AS, Morey AF, Aviles R, Garcia CR. Anterior urethral strictures: etiology and characteristics. Urology 2005;65(06): 1055-1058

2 Lumen N, Hoebeke P, Willemsen P, De Troyer B, Pieters R, Oosterlinck W. Etiology of urethral stricture disease in the 21st century. J Urol 2009;182(03):983-987

3 Edwards LE, Lock R, Powell C, Jones P. Post-catheterisation urethral strictures. A clinical and experimental study. Br J Urol 1983;55(01):53-56

4 Kevin T, McVary H. Surgical Management of Lower Urinary Tract Symptoms Attributed to Benign Prostatic Hyperplasia (2018) [Internet]. Disponible en: http://www.auanet.org/guidelines/ surgical-management-of-lower-urinary-tract-symptomsattributed-to-benign-prostatic-hyperplasia-(2018)

5 Jeffrey A, Cadeddu M. Clinically localized prostate cancer: AUA/ASTRO/SUO Guideline 2017 [Internet]. Disponible en: file://C:/Users/unidades/Downloads/Clinically-LocalizedProstate-Cancer.pdf

6 Pigrau C. [Nocosomial urinary tract infections]. Enferm Infecc Microbiol Clin 2013;31(09):614-624

7 Zhou SK, Zhang J, Sa YL, et al. Etiology and Management of Male Iatrogenic Urethral Stricture: Retrospective Analysis of 172 Cases in a Single Medical Center. Urol Int 2016;97(04):386-391

8 Simhan J. Reducing Iatrogenic Urethral Trauma. J Urol 2015;194 (04):871-872

9 Thomas AZ, Giri SK, Meagher D, Creagh T. Avoidable iatrogenic complications of urethral catheterization and inadequate intern training in a tertiary-care teaching hospital. BJU Int 2009;104 (08):1109-1112

10 Kashefi C, Messer K, Barden R, Sexton C, Parsons JK. Incidence and prevention of iatrogenic urethral injuries. J Urol 2008;179(06): 2254-2257, discussion 2257-2258

11 Talja M, Korpela A, Järvi K. Comparison of urethral reaction to full silicone, hydrogen-coated and siliconised latex catheters. Br J Urol 1990;66(06):652-657

12 Elliot S, Brandes S. Etiology, Histology, and Classification of Urethral Stricture Disease. Current Clinical Urology 2013:95-102

13 Katsumi HK, Kalisvaart JF, Ronningen LD, Hovey RM. Urethral versus suprapubic catheter: choosing the best bladder management for male spinal cord injury patients with indwelling catheters. Spinal Cord 2010;48(04):325-329

14 Weld KJ, Dmochowski RR. Effect of bladder management on urological complications in spinal cord injured patients. J Urol 2000;163(03):768-772

15 Tao H, Jiang YY, Jun Q et al. Analysis of risk factors leading to postoperative urethral stricture and bladder neck contracture following transurethral resection of prostate. Int Braz J Urol 2016; 42(02):302-311

16 Rassweiler J, Teber D, Kuntz R, Hofmann R. Complications of transurethral resection of the prostate (TURP)-incidence, management, and prevention. Eur Urol 2006;50(05):969-979, discussion 980

17 Sikafi Z, Butler MR, Lane V, O'Flynn JD, Fitzpatrick JM. Bladder neck contracture following prostatectomy. Br J Urol 1985;57(03): 308-310

18 Greene LF, Robinson HP. Postoperative contracture of the vesical neck. V. Clinical findings, symptoms and diagnosis J Urol 1965; 94:141-147

19 Cruz García-Villa P, Schroede-Ugalde M, Landa Soler-Martín M, Mendoza-Peña F. Factores de riesgo para el desarrollo de estenosis de uretra en pacientes operados de resección transuretral de próstata. Rev Mex Urol 2013;73(04):166-174

20 Sciarra A, Salciccia S, Albanesi L, Cardi A, D’Eramo G, Di Silverio F. Use of cyclooxygenase-2 inhibitor for prevention of urethral strictures secondary to transurethral resection of the prostate. Urology 2005;66(06):1218-1222
21 Tasci AI, Tugcu V, Sahin S, Zorluoglu F. Rapid communication: photoselective vaporization of the prostate versus transurethral resection of the prostate for the large prostate: a prospective nonrandomized bicenter trial with 2-year follow-up. J Endourol 2008;22(02):347-353

22 Al-Ansari A, Younes N, Sampige VP, et al. GreenLight HPS 120-W laser vaporization versus transurethral resection of the prostate for treatment of benign prostatic hyperplasia: a randomized clinical trial with midterm follow-up. Eur Urol 2010;58(03): 349-355

23 Elzayat EA, Habib EI, Elhilali MM. Holmium laser enucleation of the prostate: a size-independent new "gold standard". Urology 2005;66(5, Suppl)108-113

24 Krambeck AE, Handa SE, Lingeman JE. Experience with more than 1,000 holmium laser prostate enucleations for benign prostatic hyperplasia. J Urol 2013;189(1, Suppl)S141-S145

25 Varkarakis I, Kyriakakis Z, Delis A, Protogerou V, Deliveliotis C. Long-term results of open transvesical prostatectomy from a contemporary series of patients. Urology 2004;64(02):306-310

26 Campos-Juanatey F, Portillo Martín JA, Gómez Illanes R, Velarde Ramos L. Nontraumatic posterior urethral stenosis. Actas Urol Esp 2017;41(01):1-10

27 American Urological Association - Surgical Management of Stones. AUA/Endourology Society Guideline [Internet]. [citado 2 de junio de 2018]. Disponible en: http://www. auanet.org/guidelines/surgical-management-of-stones(aua/endourological-society-guideline-2016)

28 Kau E, Ng C, Fuchs G. Complications of ureteroscopic surgery. En: Taneja S, editor. Complications of Urologic Surgery. $4^{\text {th }}$ ed. USA: W.B. Saunders; 2010:303-316

29 Osther PJS. Risks of flexible ureterorenoscopy: pathophysiology and prevention. Urolithiasis 2018;46(01):59-67

30 Sorokin I, Mamoulakis C, Miyazawa K, Rodgers A, Talati J, Lotan Y. Epidemiology of stone disease across the world. World J Urol 2017;35(09):1301-1320

31 Cancer Statistics Review. 1975-2015 - SEER Statistics [Internet] [citado 2 de junio de 2018]. Disponible en: https://seer. cancer.gov/csr/1975_2015/

32 Professionals S-O. Prostate Cancer [Internet]. Uroweb [citado 2 de junio de 2018]. Disponible en: https://uroweb.org/guideline/ prostate-cancer/?type=archive

33 Erickson BA, Meeks JJ, Roehl KA, Gonzalez CM, Catalona WJ. Bladder neck contracture after retropubic radical prostatectomy: incidence and risk factors from a large singlesurgeon experience. BJU Int 2009;104(11):1615-1619

34 Westney OL. Salvage surgery for bladder outlet obstruction after prostatectomy or cystectomy. Curr Opin Urol 2008;18(06): 570-574

35 Hofer MD, Liu JS, Morey AF. Treatment of Radiation-Induced Urethral Strictures. Urol Clin North Am 2017;44(01):87-92

36 Ishiyama $\mathrm{H}$, Hirayama $\mathrm{T}$, Jhaveri $\mathrm{P}$, et al. Is there an increase in genitourinary toxicity in patients treated with transurethral resection of the prostate and radiotherapy? A systematic review. Am J Clin Oncol 2014;37(03):297-304

37 Browne BM, Vanni AJ. Management of Urethral Stricture and Bladder Neck Contracture Following Primary and Salvage Treatment of Prostate Cancer. Curr Urol Rep 2017;18(10):76

38 Elliott SP, Meng MV, Elkin EP, McAninch JW, Duchane J, Carroll PRCaPSURE Investigators. Incidence of urethral stricture after primary treatment for prostate cancer: data From CaPSURE. J Urol 2007;178(02):529-534, discussion 534

39 Mohammed N, Kestin L, Ghilezan M, et al. Comparison of acute and late toxicities for three modern high-dose radiation treatment techniques for localized prostate cancer. Int J Radiat Oncol Biol Phys 2012;82(01):204-212

40 Lukka H, Waldron T, Chin J, et al;Genitourinary Cancer Disease Site Group of Cancer Care Ontario's Program in Evidence-Based Care. 
High-intensity focused ultrasound for prostate cancer: a systematic review. Clin Oncol (R Coll Radiol) 2011;23(02):117-127

41 Warmuth M, Johansson T, Mad P. Systematic review of the efficacy and safety of high-intensity focussed ultrasound for the primary and salvage treatment of prostate cancer. Eur Urol 2010;58(06): 803-815

42 Hamdy FC, Donovan JL, Lane JA, et al;ProtecT Study Group. 10-Year Outcomes after Monitoring, Surgery, or Radiotherapy for Localized Prostate Cancer. N Engl J Med 2016;375(15):1415-1424

43 Breyer BN, Davis CB, Cowan JE, Kane CJ, Carroll PR. Incidence of bladder neck contracture after robot-assisted laparoscopic and open radical prostatectomy. BJU Int 2010;106(11):1734-1738

44 Orvieto MA, Zorn KC, Gofrit ON, Anderson J, Zagaja GP, Brendler CB. Surgical modifications in bladder neck reconstruction and vesicourethral anastomosis during radical retropubic prostatectomy to reduce bladder neck contractures. Can J Urol 2006;13(06):3353-3357

45 Zelefsky MJ, Chan H, Hunt M, Yamada Y, Shippy AM, Amols H. Long-term outcome of high dose intensity modulated radiation therapy for patients with clinically localized prostate cancer. J Urol 2006;176(4 Pt 1):1415-1419
46 Safiullah S, Lama DJ, Patel R, Clayman RV. Procedural Module: Flexible Cystoscopy. J Endourol 2018;32(S1):S2-S6

47 Terlecki RP, Steele MC, Valadez C, Morey AF. Urethral rest: role and rationale in preparation for anterior urethroplasty. Urology 2011; 77(06):1477-1481

48 Mori RL, Angermeier KW. Staged urethroplasty in the management of complex anterior urethral stricture disease. Transl Androl Urol 2015;4(01):29-34

49 Boswell TC, Hebert KJ, Tollefson MK, Viers BR. Robotic urethral reconstruction: redefining the paradigm of posterior urethroplasty. Transl Androl Urol 2020;9(01):121-131

50 Tabei T, Horiguchi A, Kobayashi K. Complicated bulbar urethral stricture successfully treated using augmented anastomotic urethroplasty: A case report. IJU Case Rep 2019;2(05):292-295

51 Steenkamp J, Heyns C, De Kock M. Outpatient treatment for male urethral strictures-dilatation versus internal urethrotomy. S Afr J Surg 1997;35(03):125-130

52 Heyns C, Steenkamp J, de Kock M, Whitaker P. Treatment of male urethral strictures: is repeated dilation or internal urethrotomy useful? J Urol 1998;160(02):356-358 